\title{
Executive attention and task switching in category learning: Evidence for stimulus-dependent representation
}

\author{
Michael A. Erickson \\ University of California, Riverside, California
}

\begin{abstract}
One class of multiple-system models of category learning posits that within a single category-learning task people can learn to utilize different systems with different category representations to classify different stimuli. This is referred to as stimulus-dependent representation (SDR). The use of SDR implies that learners switch from subtask to subtask as trials demand. Thus, the use of SDR can be assessed via slowed response times, following a representation switch. Additionally, the use of SDR requires control of executive attention to keep inactive representations from interfering with the current response. Subjects were given a category learning task composed of one- and two-dimensional substructures. Control of executive attention was measured using a working memory capacity (WMC) task. Subjects most likely to be using SDR showed greater slowing of responses following a substructure switch and a greater correlation between learning performance and WMC. These results provide support for the principle of SDR in category learning and the reliance of SDR on executive attention.
\end{abstract}

As people go through life, they are often called upon to learn new classifications. Some classifications, such as "low carb," tend to be learned rapidly, whereas others, such as "baseball hit so that an outfielder should charge it," tend to be learned more gradually. Category learning researchers have theorized that people utilize multiple systems to learn new classifications, and that these systems are suited to learning different types of classifications at different rates (e.g., Ashby, Alfonso-Reese, Turken, \& Waldron, 1998; Erickson \& Kruschke, 1998, 2002; Smith, Patalano, \& Jonides, 1998). According to these multiple-system accounts of category learning, when a category can be defined by a small number of criteria that use single psychological dimensions, it is likely to be learned by a system using rule-based category representation; and when a category requires the combination of information from multiple psychological dimensions, it is likely to be learned by a system using similarity-based category representation.

Different theories have been proposed to account for how people select which system to use. One class of theories holds that, over the course of learning, people identify which system is best suited for the current task. Because these theories learn to select a single system with a single category representation for all stimuli, they are referred to in this article as utilizing stimulus-independent representation. Another class of theories holds that people identify which system is best suited for different stimuli within the task. Because these theories can select different classification systems with their own category representation as a function of the current stimulus, they are referred to in this article as utilizing stimulus-dependent representation. This article tests predictions made by this latter class.

Because stimulus-dependent theories of category learning posit that people can learn to use different classification systems to classify the stimuli for which they are best suited, these systems may be thought of as "experts" that learn to classify a subset of the stimuli. A classifier that utilizes stimulus-dependent representation can have a functional advantage, because it can learn to use its representations strategically to partition complex classification tasks into simpler ones that place reduced demands on limited-capacity attention. Evidence of task partitioning has been shown in category learning and in function learning (Erickson, 1999; Erickson \& Kruschke, 1998, 2002; Kalish, Lewandowsky, \& Kruschke, 2004; Kruschke \& Erickson, 1994; Lewandowsky, Kalish, \& Ngang, 2002; Lewandowsky \& Kirsner, 2000; Lewandowsky, Roberts, \& Yang, 2006; Yang \& Lewandowsky, 2003, 2004). Task partitioning, however, comes at a cost. The process of dividing a task into subtasks is an additional learning task. Moreover, once an overall task has been divided, the classification system must engage in a two-step process on each trial: First, the correct expert must be chosen; second, that expert must be used to perform the classification.

Erickson and Kruschke $(1998,2002)$ have previously found support for stimulus-dependent representation by examining how subjects generalize beyond the range of training in rule-and-exception category structures (see also Denton, Kruschke, \& Erickson, in press). The model they used to account for people's task partitioning behavior is

M.A. Erickson, erickson@ucr.edu 
called ATRIUM. ${ }^{1}$ ATrium accounts for task partitioning by positing at least two category learning modules that serve as different kinds of experts, along with a gating mechanism that learns to partition the stimulus space so that each expert is responsible for the classification of a subset of the stimuli. In this article, I test two hypotheses. The first is that people can perform category learning tasks using task partitioning. The second is that when a category learning task is partitioned, learners rely on executive attention to select and maintain the task goal on each trial. Executive attention has been characterized as having the role of maintaining a representation of the stimulus, context, and task goals in the presence of interference (Engle \& Kane, 2004; Kane \& Engle, 2002; Norman \& Shallice, 1986). In models such as ATRIUM, the gating mechanism maintains the task goal on each trial by permitting the output from one representation to influence the final response while keeping the remaining representations from doing so, and by channeling feedback to the representation best suited to use it.

This article examines executive attention from an individual-differences perspective. A basic premise is that most classification problems that admit task partitioning also admit single-system strategies (e.g., Erickson, 1999; Yang \& Lewandowsky, 2003, 2004). This means that subjects in category learning experiments self-select whether to approach a classification task in a unitary manner or via partitioning. Figure 1 shows an overview of the variables under investigation in this article. Whether or not subjects use partitioning, and hence, stimulus-dependent representation, is shown as the latent variable, "SDR Yes/No." Four other variables are measured: subjects' accuracy during learning; their working memory span; the response time (RT) cost involved in switching from one category substructure to another; and the number of different types of decision bounds they employ, as measured by model fitting. Learning accuracy is commonly measured in category learning tasks. The other three variables are less common, and are therefore explained in more detail.

\section{Working Memory Capacity}

Working memory capacity (WMC) is different from short-term memory (STM) capacity. STM capacity is

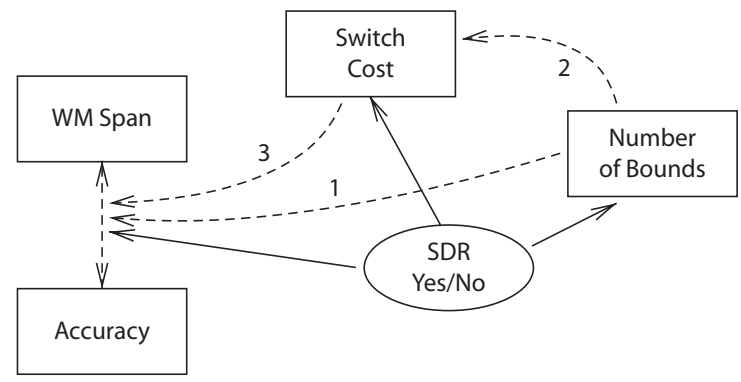

Figure 1. The variables examined in this article. Boxes indicate manifest variables, and the oval indicates a latent variable. Solid lines indicate theoretical causal relationships, and dashed lines indicate data analyses. WM, working memory; SDR, stimulusdependent representation. measured using simple span tasks, in which the subject only needs to remember the items in a memory set. WMC is measured using complex span tasks. These tasks measure working memory span under dual-task conditions that limit subjects' capacity to rehearse or elaborate the items in the memory set. In one such task, an operation-span task, each trial is comprised of (1) an equation that must be read aloud and verbally verified as true or false, and (2) a word that is to be read aloud and remembered. For example, a subject might see "Is $(7 \times 2)-6=6$ ? apple." The correct response for the equation would be "no," and the subject would need to remember "apple" along with the words following the subsequent equations. The primary dependent measure in this task is the total number of words that were recalled in correct order out of three memory sets of lengths $2-5$ [a total of $3 \cdot(2+3+4+5)=42$ words]. Accuracy on the equation verification task is also measured and is used to remove from the analysis subjects who may have been neglecting the secondary task. Other working memory tasks use sentences or visual displays in place of the equations, and letters, numbers, or visual displays in place of the words in the memory set.

Although working memory and STM span tasks may seem similar, they have quite different relationships to performance in other tasks. Individual differences in measurements of people's WMC have been found to correlate with performance on a variety of different tasks that include both high-level tasks, such as reading comprehension (Daneman \& Carpenter, 1980, 1983), vocabulary learning (Daneman \& Green, 1986), learning computer programming (Shute, 1991), Raven's advanced progressive matrices (Conway, Cowan, Bunting, Therriault, \& Minkoff, 2002; Engle, Tuholski, Laughlin, \& Conway, 1999) and verbal and quantitative SAT scores (Turner \& Engle, 1989), and low-level attentional tasks, such as dichotic listening (Conway, Cowan, \& Bunting, 2001), an antisaccade task (Kane, Bleckley, Conway, \& Engle, 2001), and a Stroop (1935) task (Kane \& Engle, 2003; Long \& Prat, 2002). This correlation with low-level attentional tasks has led theorists to postulate that measures of WMC relate to people's ability to avoid distraction from interfering information and to keep themselves from emitting prepotent responses (Engle, 2002; Engle et al., 1999). Measures of WMC appear to measure two main components: a storage component and a control component. The control component has been called executive attention by Engle and colleagues, and seems to share a number of properties with the supervisory attentional system described by Norman and Shallice (1986). One source of evidence for these two components comes from confirmatory factor analyses, in which a number of working memory and STM span tasks were completed by each subject along with measures of general fluid intelligence (gF; Engle et al., 1999). Although performance on the two types of span tasks was correlated $(r=.68)$, only performance on the working memory span task was correlated with the measures of $\mathrm{gF}$ (WMC $r=.59$ and STM $r=-.13$ ). This suggests that working memory and STM may share common storage capacities, but that working memory taps additional resources. Kane et al. (2004) per- 
formed a similar study in which they used factor analysis to separate the executive attention component of working memory span tasks from the storage components. They estimated relatively strong correlations between working memory span tasks and executive attention $(.52 \leq r \mathrm{~s} \leq$ $.79)$, and even without the storage components, executive attention still correlated strongly with gF $(r=.51)$.

In recent work, Engle and Kane (2004) emphasized the control of memory in their definition of executive attention as "the ability to maintain stimulus and response elements in active memory, particularly in the presence of events that would capture attention away from that enterprise" (pp. 192-193). A model of category learning that can utilize stimulus-dependent representation needs to rely on executive attention more when using partitioning to learn a task. This is because task partitioning requires it to maintain one category representation and block interference from other representations, so that the correct representation is used to generate the response. Therefore, a greater correlation should be found between category learning performance and measurements of WMC for subjects who partition the category learning task than for those who do not. This is shown by the arrow in Figure 1 from "SDR Yes/No" to the double-headed arrow between "WM Span" and "Accuracy."

\section{Number of Bounds}

The category structure used in this study is designed to allow for task partitioning. It is composed of two substructures used by Ashby, Ell, and Waldron (2003). In their studies, Ashby et al. (2003) manipulated the category structure between subjects. One group of subjects learned to classify items drawn from a category structure in which optimal classification depended on attention to just one dimension: I refer to this as a one-dimensional (or 1-D) category structure. The other group learned to classify items drawn from a structure in which optimal classification depended on attention to two dimensions: I refer to this as a two-dimensional (or 2-D) category structure. As is shown in Figure 2, in the present study these two structures are manipulated within subjects: All subjects were exposed to both the 1-D and the 2-D substructures. In previous experiments with similar category structures, some subjects learned to classify the overall category structure by partitioning it into substructures, and other subjects treated it as a unitary category structure (Erickson, 1999; Yang \& Lewandowsky, 2003, 2004).

The category structure used here allows for $100 \%$ accuracy, if items from the 2-D substructure are classified with the proper 2-D bound, and items from the 1-D substructure are classified with the proper 1-D bound. Nevertheless, suboptimal approaches are also possible. A likely suboptimal approach is to classify items from both substructures using horizontal 1-D bounds. Such an approach would only yield about $80 \%$ accuracy on the 2-D substructure, but, combined with possible perfect accuracy on the 1-D substructure, overall accuracy could be as high as $90 \%$. Other suboptimal sets of boundaries are possible; for example, because combining information from both dimensions is adaptive in the 2-D substructure, subjects might

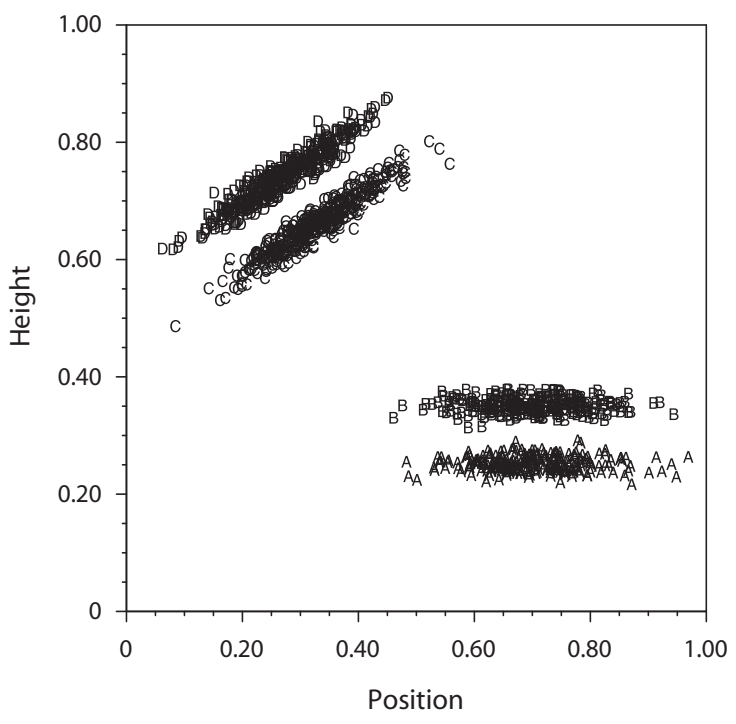

Figure 2. The category structure used in the experiment. Each letter, $\mathrm{A}, \mathrm{B}, \mathrm{C}$, or $\mathrm{D}$, in the figure indicates an item of that category presented during the experiment. Categories $A$ and $B$ comprised the 1-D substructure, and categories $C$ and $D$ comprised the 2-D substructure.

adopt a suboptimal 2-D bound for the 1-D substructure. Finally, exhausting the logical possibilities, some subjects might adopt a 1-D bound for the 2-D substructure, and a 2-D bound for the 1-D substructure.

A model of category learning that utilizes stimulusdependent representation, such as ATRIUM, would account for the classification data from these four approaches to the category structure in different ways. For the subjects who use a single bound type in the two substructures (i.e., 1-D bounds for both substructures, or 2-D bounds for both), it would account for the results using a single representation. To account for two 1-D bounds, it would use rule representation alone; and to account for two 2-D bounds, it would use similarity-based representation alone. Thus, to account for the data from subjects who use a single bound type, it would not invoke stimulus-dependent representation, but would use one representation for the entire category learning task. The converse is true for this kind of model when accounting for the data from subjects who used two bound types. To account for the data from those subjects, it would utilize rule representation for the 1-D bound and similaritybased representation for the 2-D bound; that is, when accounting for the data from these subjects, the model would utilize stimulus-dependent representation.

These accounts of the different patterns of data are the most natural for these kinds of models, but they do not necessarily match the way the data were generated. For example, even though it is most likely that the data from subjects with two bound types would be correctly modeled using multiple representations, some of the subjects could be completing the category learning task using pure similarity-based classification. This means that the arrow in Figure 1 from "SDR Yes/No" to "Number of Bounds" 
does not signify a deterministic relation between the use of stimulus-dependent representations and the number of category bounds, but a probabilistic one. This is addressed further in the General Discussion.

\section{Task Switching}

Task switching is typically investigated in experiments in which subjects are explicitly given multiple tasks to perform sequentially. In these experiments, they show costs of switching from one task to another: On trials following a task switch, subjects tend to respond more slowly than on trials in which the task has remained the same (for reviews, see Logan \& Gordon, 2001; Monsell, 2003). The RT difference between trials that require subjects to switch tasks and ones that do not is referred to as the task-switch cost. Task-switch costs have been explained as being composed of three factors: the time to retrieve and store the new task goals in working memory, the time to reprogram attentional systems based on revised task goals, and the interfering effect of past task goals on the present goal (Logan, 2004). In the present study, even though subjects are not explicitly given multiple tasks, it is hypothesized that some of the subjects will adopt a partitioning strategy that turns the overall classification task into multiple subtasks. This means that subjects who use task partitioning should show task-switch costs, and subjects who do not should not. This is represented in Figure 1 by the arrow from "SDR Yes/No" to "Task Switch Costs."

According to the logic outlined in Figure 1, if subjects who use task partitioning, and therefore, stimulusdependent representations, were analyzed separately from subjects who do not, there should be differences in (1) the number who use one rather than two category bound types in the two groups, (2) the task-switch costs, and (3) the correlation between working memory span and category learning accuracy. Because the use of stimulus-dependent representation is a latent variable that cannot be measured directly, the analyses in this article instead examine the relationships between the manifest variables. The three main analyses are indicated by dashed arrows, and the numbers in Figure 1 correspond to the numbered analyses in the following description.

Analysis 1 uses the number of category bounds as a proxy for a measurement of task partitioning. It tests whether subjects who use two different bound types (1-D/2-D or 2-D/1-D) show a greater correlation between working memory span and accuracy than do those who use a single bound type (1-D/1-D or 2-D/2-D). If task partitioning makes it more likely to use two bound types, subjects who do should show a greater correlation between working memory span and accuracy. Analysis 2 examines whether classification by number of bound types corresponds with task-switch costs. If task partitioning makes it more likely to use two bound types, then subjects who do should show greater task-switching costs. To the extent that the number of bound types does not correspond exactly to the use of stimulus-dependent representation, these first two analyses are somewhat conservative inasmuch as the groups that are identified may be mixtures of partitioning and nonpartitioning subjects. Analysis 3 examines the relationship between task-switching and the correlation between working memory span and category learning accuracy. If task partitioning increases task-switch costs and the correlation between working memory span and learning accuracy, task-switch costs should act as a moderator of the relationship between working memory span and learning accuracy; greater switch costs should therefore correspond to a greater correlation between working memory span and accuracy. Each of these three tests examine individual differences in the manner proposed by Underwood (1975), in which individual differences are used as a crucible in the construction of theories.

To summarize, the goal of this study is to evaluate two hypotheses regarding category learning: The first is that people can partition category learning tasks into subtasks; and the second is that when people partition category learning tasks, they rely on executive attention to maintain task goals and select responses. These hypotheses are evaluated by examining the relationship between four variables: number of bound types, task-switch costs, working memory span, and category learning accuracy. Subjects who partition the category learning task should tend to use two bound types, show greater task-switch costs, and have a stronger correlation between working memory span and category learning accuracy, whereas subjects who adopt a unitary approach should be more likely to use a single bound type, be less likely to show task-switch costs, and have a weaker correlation between working memory span and category learning accuracy.

\section{EXPERIMENT \\ Task Partitioning in Category Learning}

Subjects learned to classify rectangles that varied in height, in the horizontal position of an internal line segment, and in color into one of four categories. The category layout is shown in Figure 2. Items drawn from the 1-D category substructure (categories A and B) were presented in one color, and items drawn from the 2-D category substructure (categories $\mathrm{C}$ and $\mathrm{D}$ ) were presented in another. To facilitate learning, only items from the 1-D substructure were presented in the first phase of learning; only items from the 2-D substructure were presented in the second phase; and items from both substructures were combined in the third phase.

Following the category learning task, subjects completed an automated symmetry span task (Engle, 2005). This task measured working memory span by having subjects keep track of the positions of filled cells displayed sequentially in a grid with a secondary task of judging whether or not displays composed of filled cells in a grid possessed symmetry about the vertical axis. Although this task might seem to measure a different capacity from the one measured by the working memory span task (the operation span task) described previously, Kane et al. (2004) have provided evidence that both working memory span tasks capture the domain-general capacity to control executive attention that is the focus of this research. In particular, they estimated a correlation of $r=.73$ between the symmetry span task and executive attention (see also Engle \& Kane, 2004; Engle et al., 1999; Kane \& Engle, 2002). 


\section{Method}

Subjects

One hundred seventy undergraduate students from the University of California, Riverside, participated for research credit in an introductory psychology course. Subjects also received $\$ 5$ compensation if they obtained at least $70 \%$ correct within each category in the final block of training. Subjects reported that they had normal or corrected-to-normal visual acuity and did not have color blindness. No WMC information was recorded for 4 of the subjects due to a technical malfunction, so their data were discarded.

\section{Stimuli and Stimulus Generation}

The stimuli were fixed-width rectangles with internal verticalline segments. The rectangles varied along two dimensions: height and line segment position. The bottom and sides of the rectangles were white. The internal line segments and the top of the rectangles were either cyan or magenta. Stimuli from categories A and B were drawn with one of these colors and stimuli from categories $\mathrm{C}$ and $\mathrm{D}$ were drawn with the other. The assignment of colors to category pairs was randomized between subjects. Following Ashby et al. (2003), rectangle heights and line segment positions were selected using Ashby and Gott's (1988) stimulus randomization technique. Under this technique, each category is defined by a distribution with a given mean and variance for each dimension and covariance between dimensions. Ashby et al. (2003) used distributions that yielded $d^{\prime}=(420-280) / \sqrt{ } 330=7.7067$ when projected onto the axis connecting the means. The distributions used in the present experiment yielded this same value of $d$ ' The parameter values selected for the four categories are displayed in Table 1 as proportions of maximum possible displacement on the screen along each dimension. The maximum possible displacement for the rectangle height was 538 pixels and for the line segment position was 478 pixels. Thus, for example, the mean line segment position for category A was $0.70 \times 478$ pixels $=334.6$ pixels. The covariance in this experiment was chosen to make the optimal bound separating categories $\mathrm{C}$ and $\mathrm{D}$ at a $45^{\circ}$ angle above horizontal in psychological space. Erickson and Kruschke (1998) found that changes along the segment position dimension were approximately 1.5 times more salient than were changes along the height dimension. This translates to a bound that is approximately $33.7^{\circ}$ above horizontal in physical space. Accordingly, the best unidimensional rule for classifying these stimuli yielded approximately $80 \%$ correct. The resulting category structure is shown in Figure 2.

\section{Procedure}

Subjects performed the experiment in dimly lit individual cubicles. Up to 5 subjects could complete the experiment simultaneously. All subjects completed the category learning task first and the automated symmetry span task second.

Category learning task. Within the category learning task, there were 19 blocks divided into three phases. The stimuli in each block were selected and ordered randomly for each subject, given the following constraints. Each block in the first two phases contained 50 trials. In the first phase, subjects were trained on the 1-D substruc- ture. It was composed of 4 blocks in which 25 stimuli sampled from category A and 25 stimuli sampled from category B were presented. In the second phase, subjects were trained on the 2-D substructure. It was composed of 11 blocks in which 25 stimuli sampled from category $\mathrm{C}$ and 25 stimuli sampled from category $\mathrm{D}$ were presented. The third phase contained 4 blocks in which stimuli from both substructures were randomly intermixed. Each block in this phase contained 100 trials and was composed of 25 stimuli sampled from each of the four categories.

The four categories were randomly assigned different category labels selected from the letters A, S, K, and L, subject to the constraint that within each substructure one label was selected from the letters $\mathrm{A}$ and $\mathrm{S}$, and the other label was selected from the letters $\mathrm{K}$ and L. Subjects were instructed to use the left hand to make A and S responses and the right hand to make $\mathrm{K}$ and $\mathrm{L}$ responses. The experimenter checked to verify hand positions 3-4 times over the course of the experimental session. This was done to match the procedure of Ashby et al. (2003) as closely as possible.

Subjects were told that the experiment was composed of multiple tasks and that the first task investigated how people learn to classify space shuttle schematics. They were told that if they obtained at least $70 \%$ correct on all four categories in the final block of training, they would receive a $\$ 5$ bonus. They were also informed that the schematics were composed of a rectangle whose height signified the height of the shuttle and a vertical line segment whose position signified the door position. Before the first phase, they were told that they only needed to attend to the height to classify the stimuli correctly. Before the second phase, they were told that they needed to attend to both the height and the line segment position. They were given no additional instructions before the third phase, when they began classifying stimuli from all four categories. Subjects were given no instructions regarding the color of the stimuli.

Symmetry span task. Following the category learning task, subjects completed the automated symmetry span task (Engle, 2005). This task was adapted from the symmetry span task described by Kane et al. (2004). This task consisted of two main parts: a practice session and the actual WMC assessment. The practice session was itself composed of three parts.

In the practice session, subjects were first exposed to the sequence task. In this practice task, they were presented with a sequence of black $4 \times 4$ grids on a white background. In each grid, a different cell was colored red. Each grid was presented for $650 \mathrm{msec}$, with 500 -msec intervals between grids. After each sequence, subjects were required to recall the locations of the colored cells in order. They gave their responses using the computer mouse by clicking cells in a grid in the order in which they had seen those cells filled. These responses were untimed. In this portion of the practice task, they saw two series of set size 2 and of set size 3, where set size indicates the number of filled cells in the sequence.

Next, subjects were given the practice symmetry task. In each trial in this task, they were presented with a black $8 \times 8$ grid, in which some of the cells were colored black. The grid was presented on a white background. The subjects were required to determine whether the colored cells showed left-right symmetry. Once they had decided, they clicked the mouse to proceed to a response display.

Table 1

Distribution Parameter Values Used in the Generation of the Stimuli in Each Category Structure

\begin{tabular}{lllllll}
\hline $\begin{array}{c}\text { Category Type/ } \\
\text { Label }\end{array}$ & \multicolumn{2}{c}{ Means } & & \multicolumn{2}{c}{ Variance } & \\
\cline { 2 - 3 } & Position & Height & & Position & Height & Covariance \\
\hline $\begin{array}{l}\text { Unidimensional } \\
\text { A }\end{array}$ & & & & & & \\
B & .70000 & .25000 & & .0071429 & .0001684 & .0000000 \\
Diagonal & .70000 & .35000 & .0071429 & .0001684 & .0000000 \\
C & & & & & & \\
D & .32774 & .65840 & .0049969 & .0023144 & .0032190 \\
\hline
\end{tabular}


They gave their response by clicking a "yes" or a "no" box. This was followed by accuracy feedback. They saw 15 symmetry trials in the symmetry task. The practice symmetry task served to familiarize subjects with the task itself and to measure how long each subject took to evaluate symmetry. Subjects' mean RT, plus $2.5 S D$, was used as a deadline for the symmetry portion of the WMC assessment. Adjusting the deadline according to individual subjects' practice RTs was used to help account for individual differences in the assessment, so that faster subjects could not use extra time to compensate for interference from the secondary task.

The final practice task combined the sequence and the symmetry tasks by interleaving the memory sequences and the symmetry judgments. Each item in each sequence was preceded by a symmetry judgment trial. This final practice task was the same as the WMC assessment that followed. In the final practice task, and in the WMC assessment, accuracy on the symmetry portion was emphasized, and subjects were told that they needed to obtain $85 \%$ correct. Subjects were also informed that they would need to respond as quickly on the symmetry task as they had during practice or the trial would count as an error. Subjects completed three final practice sequences of set size 2 . This was followed by a brief set of instructions reminding them of the importance of maintaining symmetry accuracy of at least $85 \%$, and then by the WMC assessment itself, which consisted of three sequences of each of the set sizes 2, 3, 4, and 5, yielding 12 sets in total. The primary dependent measure was the number of cell locations correctly recalled in order. This could vary between 0 and $3 \cdot(2+3+4+5)=42$.

\section{Results and Discussion}

As is shown in Figure 1, three main analyses were performed. Preparatory to performing these analyses, subjects were grouped using a model-based analysis according to the kinds of bounds they used to classify stimuli from the two category substructures. Following this, the relation between subjects' category bounds and the correlation between working memory and accuracy during learning was examined, as was the relation between subjects' category bounds and switch costs. The final analysis examined the relation between switch costs and the correlation between working memory and accuracy during learning.

The analyses depend on estimates of working memory span for each subject. Following Kane et al. (2004), the inclusion criterion for these subjects was based on the secondary task performance in the WMC task and required that performance on the symmetry-judgment portion of the task was $85 \%$ correct or better. Therefore, these analyses include the 123 subjects who met this criterion.

\section{Strategy Identification}

To determine the nature of subjects' category bounds, their patterns of responses were analyzed using the general recognition theory (GRT; Ashby \& Townsend, 1986), a multidimensional generalization of signal detection theory (Green \& Swets, 1966). The GRT was used to fit boundaries with different orientations to each subject's classification responses from the last block of training (100 trials), and subjects were then categorized according to the orientation of the best-fitting bounds. The GRT models that were fit instantiated linear 1-D and 2-D boundaries, and the fits were compared using the AIC (Akaike, 1974) as the measure of lack of fit. ${ }^{2}$

Each subject's responses from each substructure were fit separately. Three models were fit to the 2-D substruc- ture: a 2-D bound, a horizontal 1-D bound, and a vertical 1-D bound. Two models were fit to the 1-D substructure: a 2-D bound and a horizontal 1-D linear bound. Subjects were classified as utilizing a 1-D or 2-D bound for the $1-\mathrm{D}$ substructure, and a 1-D or 2-D bound for the 2-D substructure, by selecting the model that yielded the lowest value of the fit statistic for each substructure. The classification data and best-fitting bounds from 4 representative subjects are shown in Figure 3. As described previously, subjects were classified into one of four groups. The first group in Table 2 (1-D/1-D) consisted of subjects who used 1-D bounds for both substructures. The subjects in the second group (1-D/2-D) used bounds that matched the substructures: a 2-D bound for the 2-D substructure, and a 1-D bound for the 1-D substructure. The third group (2-D/1-D) selected mismatched bounds: a 1-D bound for the 2-D substructure, and a 2-D bound for the 1-D substructure. The last group (2-D/2-D) consisted of subjects who used 2-D bounds for both substructures. Because models of category learning that utilize stimulusdependent representation would tend to use a singlesystem approach to data from the 1-D/1-D and 2-D/2-D groups, the 56 subjects in these two groups were classified as each utilizing a single representation type. Similarly, because these models would tend to account for the data from the 1-D/2-D and 2-D/1-D group, using rule representation for stimuli from the substructure best fit by a 1-D bound and similarity-based representation for stimuli from the substructure best fit by the 2-D bound, the 67 subjects in these two groups were classified as using two different representation types.

Table 2 shows the mean overall accuracy during learning and working memory spans for subjects in each of the four groups. Although analysis of these variables does not bear on the hypotheses under investigation, they are examined briefly to characterize the nature of the groups' performance. A $2 \times 2$ between-subjects ANOVA was performed on both dependent variables, in which the independent variables were whether subjects used a 1-D or a 2-D bound for the 1-D substructure, and whether they used a 1-D or a 2-D bound for the 2-D substructure. As might be expected, the analysis of overall accuracy indicated that subjects who had used 2-D bounds to classify items from the 2-D substructure performed better than did subjects who had used 1-D bounds $[F(1,119)=7.68$, $\left.M S_{\mathrm{e}}=0.0094, p=.0065, d=0.52\right]$. Accuracy was not affected reliably by the type of bound used to classify items from the 1-D substructure $\left[F(1,119)=1.06, M S_{\mathrm{e}}=\right.$ $0.0094, p=.31, d=0.19]$. The reason for this can be seen in Figure 3. Subjects who had used 1-D bounds to classify the 2-D substructure misclassified many more items, even in the final block of training, than did subjects who had used 2-D bounds to classify the 1-D substructure. The interaction between the two factors also failed to reach significance $\left[F(1,119)=0.29, M S_{\mathrm{e}}=0.0094, p=.59\right.$, $d=0.10]$.

Theoretically, the relation between the group assignment and working memory span seems more interesting. One might expect subjects with greater WMC to make greater use of the task partitioning strategy. However, this 
was not borne out by these analyses. Neither the main effect of bound type for the 1-D substructure $[F(1,119)=$ $\left.0.0387, M S_{\mathrm{e}}=82.53, p=.84, d=0.04\right]$, nor the effect of bound type for the 2-D substructure $[F(1,119)=0.4363$, $\left.M S_{\mathrm{e}}=82.53, p=.51, d=0.12\right]$, nor their interaction $\left[F(1,119)=0.1895, M S_{\mathrm{e}}=82.53, p=.66, d=0.08\right]$, was significant. Thus, these results failed to provide evidence that the measure of working memory span provided by the symmetry span task predicts whether or not people will approach a category learning task using a partitioning strategy.

\section{WMC and Learning}

To permit evaluation of different theories of working memory utilization in category learning, four correlations, shown in Table 2, were computed for each group. The most important correlations for evaluating predictions based on models that include stimulus-dependent representation are those listed in the first column of the correlations ("Acc./WM Span"). These show the overall correlation between subjects' working memory span obtained using the symmetry span task and their overall performance on the category learning task. The remaining correlations are not of primary interest for testing these predictions, but are useful for testing alternate theories of working memory in category learning. The second and third columns of correlations, respectively, show the correlations between subjects' working memory span and category learning performance for the 1-D and 2-D substructures. The fourth column of correlations shows the correlations between subjects' category learning performance for 1-D and 2-D substructures. This column is necessary to test the differences between the 1-D Acc./WM Span and 2-D Acc./WM Span correlations, but is not necessarily of any theoretical interest on its own (Meng, Rosenthal, \& Rubin, 1992).

If classification using task partitioning relies on executive attention, subjects who use two bound types should show a greater correlation between working memory span and learning accuracy. An examination of the first column of correlations in Table 2 indicates that, consistent with this prediction, when subjects were classified as using two different representations, the correlation between working memory span and learning performance was significantly different from 0 , but not when subjects were classified as using a single representation. The $95 \%$ confidence inter-
$1-D / 1-D(n=45)$

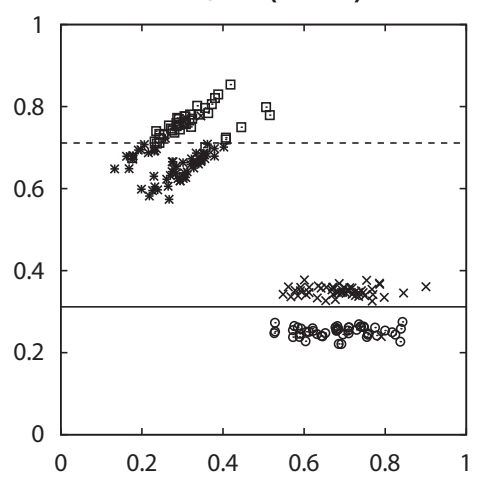

2-D/1-D $(n=16)$

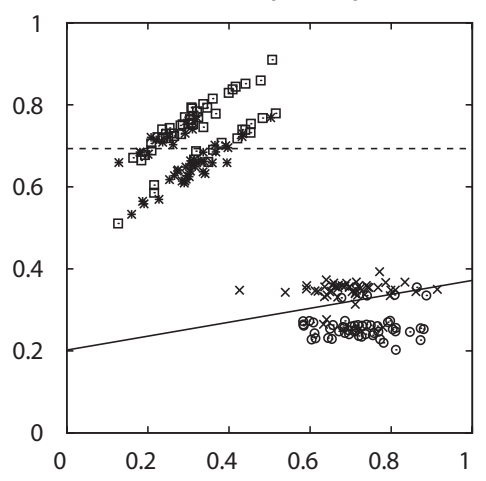

$1-D / 2-D(n=51)$

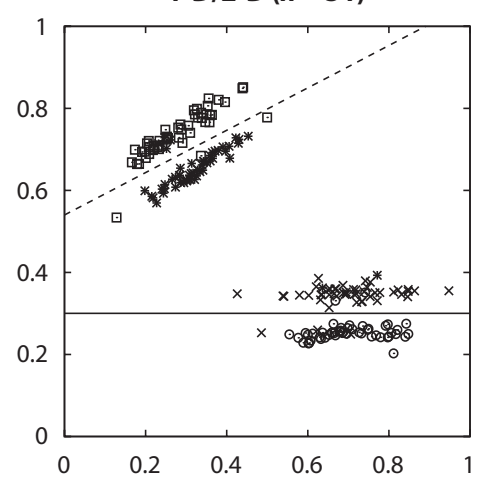

2-D/2-D ( $n=11)$

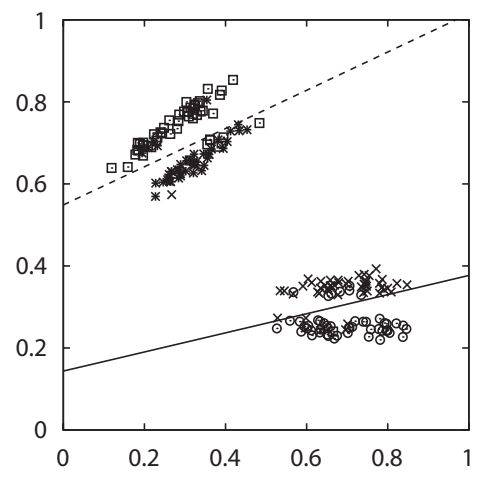

Figure 3. Data from Block 19 for 4 subjects and the corresponding best-fitting bounds. The label on each panel indicates the type of bound used for the 1-D and 2-D substructures, respectively. Category $A$ responses are indicated with a circle; category $B$, with an $\mathrm{X}$; category $C$, with an asterisk; and category $D$, with a square. 
Table 2

Descriptive Statistics and Correlations for Working Memory Capacity and Learning Performance

\begin{tabular}{|c|c|c|c|c|c|c|c|c|c|}
\hline \multirow[b]{3}{*}{ Fit Group } & \multirow[b]{3}{*}{$n$} & \multicolumn{4}{|c|}{ Mean } & \multicolumn{4}{|c|}{ Correlation } \\
\hline & & \multicolumn{2}{|c|}{ Accuracy } & \multicolumn{2}{|c|}{ WM Span } & \multirow{2}{*}{$\begin{array}{c}\text { Acc./ } \\
\text { WM Span }\end{array}$} & \multirow{2}{*}{$\begin{array}{l}\text { 1-D Acc./ } \\
\text { WM Span }\end{array}$} & \multirow{2}{*}{$\begin{array}{l}\text { 2-D Acc./ } \\
\text { WM Span }\end{array}$} & \multirow{2}{*}{$\begin{array}{l}\text { 1-D Acc. } \\
\text { 2-D Acc. }\end{array}$} \\
\hline & & $M$ & $S D$ & $M$ & $S D$ & & & & \\
\hline 1-D/1-D & 45 & .63 & .12 & 20.87 & 9.59 & .087 & -.003 & .158 & $.742^{*}$ \\
\hline $1-\mathrm{D} / 2-\mathrm{D}$ & 51 & .76 & .08 & 19.22 & 8.62 & $.300^{*}$ & $.301^{*}$ & .227 & $.554^{*}$ \\
\hline 2-D/1-D & 16 & .68 & .11 & 23.81 & 9.64 & $.686^{*}$ & $.630^{*}$ & $.562^{*}$ & $.507^{*}$ \\
\hline $2-D / 2-D$ & 11 & .78 & .05 & 23.91 & 8.13 & .230 & .311 & .070 & .333 \\
\hline Overall & 123 & .71 & .11 & 20.84 & 9.14 & .173 & .171 & .151 & $.718^{*}$ \\
\hline
\end{tabular}

Note-WM Span, working memory span. Accuracy and Acc. indicate overall learning performance averaged across both substructures. 1-D Acc. indicates learning performance for the 1-D substructure. 2-D Acc. indicates learning performance for the 2-D substructure. $\quad$ " $p<.05$.

val for the 1-D/1-D subjects' correlation $(-.2118 \leq r \leq$ .3712 ), and for that of the 2-D/2-D subjects $(-.4295 \leq r \leq$ .7290 ), both included 0 within wide margins, whereas neither that of the 1-D/2-D nor that of the 2-D/1-D subjects did (.0269 $\leq r \leq .5318$ and $.2881 \leq r \leq .8818$, respectively).

These data, then, match the theories' qualitative predictions perfectly. In addition to this inspection of individual correlation coefficients, the collection of correlation coefficients can be analyzed jointly with respect to different theories using meta-analytic procedures by selecting contrast weights that correspond to the theories' respective predictions (Rosenthal, 1991). This technique can be more conservative than merely considering which correlations are significant and which are not, because failures to reject the null can still count against the overall hypothesis. Here, the overall hypothesis - according to theories that utilize executive attention for stimulus-dependent representation - is that working memory span should correlate with learning performance for subjects classified as using two representations, and that it should not correlate with learning performance for those classified as using a single representation. The contrast that corresponds to this hypothesis tests whether the average correlation for subjects who used two representations $(M=.5190)^{3}$ is higher than that for subjects who used a single representation $(M=.1591)$. A test of this hypothesis was significant $(Z=1.6691, p=.0476$, $d=0.30$ ). When considered together with the individual analyses of the correlations presented in the first column of correlations in Table 2, this result provides evidence that executive attention played a greater role in processing for subjects who used two representations than it did for those who used just a single representation.

To provide a basis for comparison, the evidence for the predictions of models that allow for task partitioning can be contrasted with the predictions of a second hypothesis, which is that working memory span should be correlated with 1-D-learning performance. This hypothesis is suggested by theories that assume that the principal role for working memory in category learning is in the formation of 1-D category bounds (e.g., COVIS, which stands for competition between verbal and implicit systems; Ashby et al., 1998). Under such a theory, the correlation between working memory span and mean learning performance should be strongest for subjects who used 1-D bounds for both substructures, weakest for subjects who used 2-D bounds for both substructures, and at an intermediate level for subjects who used mixed representations; in other words, the correlations in the Acc./WM Span column should be ordered $1-\mathrm{D} / 1-\mathrm{D}>(1-\mathrm{D} / 2-\mathrm{D} \approx 2-\mathrm{D} / 1-\mathrm{D})>2-\mathrm{D} / 2-\mathrm{D}$. The contrast that corresponds to this hypothesis focuses on the difference between the 1-D/1-D and 2-D/2-D conditions in the Acc./WM Span column. In the data, however, the difference between the correlations is small and in the wrong direction (i.e., $1-\mathrm{D} / 1-\mathrm{D}<2-\mathrm{D} / 2-\mathrm{D})(Z=-0.3793, p=$ $.6478, d=-0.07)$. Because correlations were computed for the two substructures separately (shown in the columns labeled 1-D Acc./WM Span and 2-D Acc./WM Span), another prediction based on this same hypothesis can be tested. If working memory span is correlated with 1-D- but not 2-D-learning performance, this correlation should be greater for a substructure classified using a 1-D representation than for one classified using a 2-D representation. This entails that for the 1-D/2-D group, the 1-D Acc./WM Span correlation should be greater than the 2-D Acc./WM Span correlation, vice versa for the 2-D/1-D group, and for the 1-D/1-D and 2-D/2-D groups, the correlations in these two columns should be the same. Because the correlations within each group are themselves correlated, they were tested following Meng et al. (1992), and these tests were again combined to yield weak evidence consistent with the hypothesis $(Z=0.1716, p=.4319, d=0.03)$. Because the test examining the overall correlation between accuracy and working memory span and the test examining the separate correlations between the 1-D and 2-D accuracies and working memory span provided evidence in opposite directions, when they were combined they failed to provide support for the hypothesis that the primary role of working memory in this category learning task was in the acquisition of 1 -D category bounds $(Z=-0.1469, p=.5584$, $d=-0.03)$. The results of these correlational analyses, then, suggest that executive attention plays an important role in category learning when people partition the overall classification task into subtasks and that-for this category structure, at least - executive attention does not play an important role in rule learning by itself.

\section{Task-Switch Costs}

When a category learning task is partitioned into subtasks, task-switching research suggests that a performance cost should be incurred when subjects switch from one 
subtask to another. This prediction was examined by classifying each trial in the last block of training, Block 19, as having been preceded by one from the same or the other substructure. Trials preceded by one with the same category label were excluded, because repeated responses tend to be faster than nonrepeated responses. Incorrect responses, and responses that occurred in less than $250 \mathrm{msec}(3.18 \%)$ or more than 5,000 msec (1.05\%), were excluded from analysis. As is shown in Figure 4, subjects responded more slowly when they switched substructure $(M=1,296 \mathrm{msec}, S D=$ $353 \mathrm{msec}$ ) than when they did not (but did switch response; $M=992 \mathrm{msec}, S D=290 \mathrm{msec})[F(1,120)=143.71$, $\left.M S_{\mathrm{e}}=39,222, p<.0001, d=2.19\right]$. Critically, consistent with the task-partitioning/task-switching hypothesis, the difference was smaller for subjects who were classified as using a single representation type $(M=228 \mathrm{msec}, S D=$ $340 \mathrm{msec}$ ) than for those who were classified as using two types $(M=366 \mathrm{msec}, S D=219 \mathrm{msec})[F(1,120)=7.36$, $\left.M S_{\mathrm{e}}=39,222, p=.0077, d=0.50\right]$.

Identification of a differential task-switching effect for the two groups provides evidence, to an extent, that the technique used to identify subjects' strategies correctly identified subjects who were using one rather than two representations. Nevertheless, the group of subjects identified as using a single representation did respond more slowly when they switched substructures than when they did not $[t(54)=4.98$, $p<.0001, d=1.36]$. The most likely explanation for this is that - as was suggested in the introduction - the two groups are composed of a mixture of subjects, some of whom used a single representation and some of whom used two representations. Even though these groups might be composed of mixtures, showing that subjects who use two representation types have a greater correlation between accuracy and working memory span and have greater switch costs suggests that the likelihood of being classified as using two representation types is higher for subjects who are using stimulusdependent representation than it is for subjects who are not. To the extent that these groups are composed of mixtures, however, tests of their differences are more conservative than if the groups had been identified perfectly.

\section{Task-Switch Costs and Working Memory Span}

The first two analyses performed depended on the classification of subjects as using one or two representation types. If the variables under investigation are related, as is diagrammed in Figure 1, the use of stimulus-dependent representation should simultaneously increase task-switch costs and the relation between working memory span and learning accuracy; in other words, whereas the number of category bound types is a discrete index of the use of task partitioning, task-switch costs should be a continuous index of its use. This third analysis investigated this prediction by testing whether measurements of switch cost moderate the relationship between working memory span and accuracy. Moderation is expressed in a multiple regression framework as an interaction, so the regression model of primary interest was

$$
\begin{aligned}
\widehat{\text { Acc. } .}= & B_{0}+B_{1} \cdot \mathrm{WMSpan}+B_{2} \cdot \text { Switch Cost } \\
& +B_{3} \cdot \mathrm{WMSpan} \cdot \text { Switch Cost },
\end{aligned}
$$

and $B_{3}$ was the critical regression coefficient. If $B_{3}>0$, this means that as switch cost increases, so does the correlation between working memory span and overall accuracy. Because previous analyses have shown that the number of representation types influenced accuracy, the switch cost, and the relationship between working memory span and accuracy, it was important to determine whether including the effect of the number of representation types and its interactions in the analysis improved the quality of the final regression model. To do this, the fit of several models to the data were compared. Following Cohen, Cohen, West, and Aiken (2003), the continuous predictors in all models were centered prior to being fit. The first model is shown in Equation 1, and it provided an adequate account of the data $\left[F(3,118)=9.05, p=.00002, R^{2}=\right.$ $.1870]$. The second added the main effect of number of representation types and yielded a better fit $[F(4,118)=$ $\left.10.31, p<.000001, R^{2}=.2605\right]$ ]. A comparison of the two models indicated that the difference between the two fits was significant $[F(1,117)=11.639, p=.00089]$. No further additions of two- and three-way interactions between switch cost, number of representation types, and working memory span provided significant improvement over the second model (all $F$ 's $<1$ ).

A summary of the regression analysis using the second model is shown in Table 3 . All four correlation coefficients were significant, including the critical interaction coefficient. Working memory span, switch cost, and the number of representation types used by a subject were all directly related to accuracy. Interestingly, working memory span by itself did not show a significant correlation with overall accuracy (see the bottom row of the Acc./WM Span column in Table 2), but it did as part of a model providing a more complete explanation of the variance in accuracy. Figure 5 shows the interaction be-

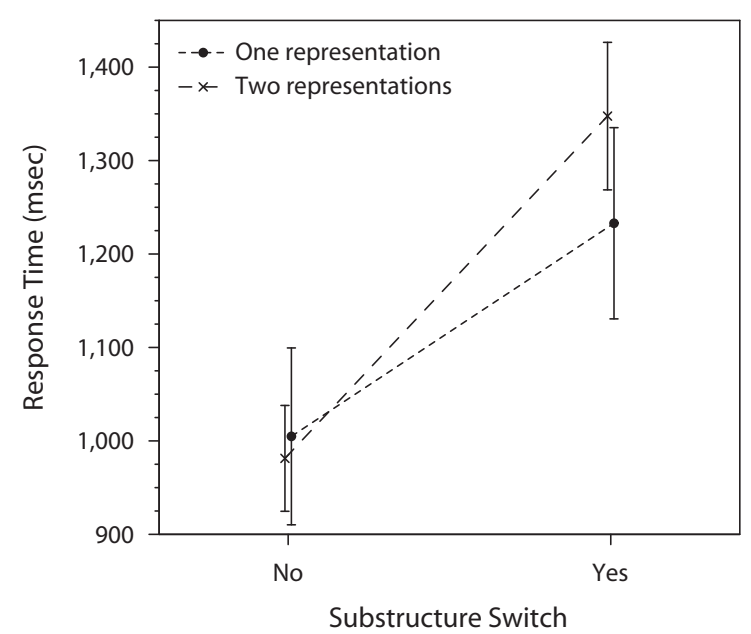

Figure 4. Response times for trials following a trial in the same substructure or one in the other substructure in Block 19, the last block of training for subjects who were assessed as using one or two representations. Error bars indicate the $\mathbf{9 5 \%}$ confidence intervals of the means. 
tween switch cost and working memory span by plotting the relation between working memory span and accuracy at three levels of switch cost. At the mean level of switch cost, learning accuracy is predicted to increase at a rate of $0.24 \%$ for every additional correctly recalled cell position in the symmetry span task. When switch cost is high (1 SD above the mean), the rate increases to $0.66 \%$, and when switch cost is low (1 SD below the mean), the rate decreases to $-0.18 \%$.

This result is consistent with the hypothesis that people can use task partitioning in category learning and that task partitioning relies on executive attention. According to this hypothesis, when subjects approach a category learning task by partitioning it into subtasks, one of the costs of that approach is that on each trial they need to determine how to classify the current stimulus and coordinate their response accordingly. If a trial requires that they switch from one subtask to another, their responses are slowed. Also, if they are using different representations for different stimuli, their ability to control executive attention, thereby keeping other category representations from interfering with the current one, should affect accuracy. For subjects who do not partition the task, switch costs should be reduced or eliminated, and the relationship between executive attention and accuracy should be reducedexactly as was found.

\section{GENERAL DISCUSSION}

The present study yielded two main results: First, some subjects approached the category learning task by partitioning it into subtasks; second, those subjects who partitioned the overall learning task relied on executive attention more than did those who did not. These two results
Table 3

Summary of Regression Analysis for Variables Predicting Overall Category Learning Accuracy

\begin{tabular}{lccc}
\hline \multicolumn{1}{c}{ Variable } & $B$ & $B(S E)$ & $\beta$ \\
\hline WM span & .00255 & .000975 & .023 \\
Switch cost & .00007 & .000032 & .020 \\
Number of representation types & .06293 & .018450 & .031 \\
Switch cost $\times$ WM span & .00001 & .000004 & .036 \\
\hline
\end{tabular}

Note-All coefficients are significant at the $\alpha=.05$ level.

were identified by examining two indicators of the use of multiple systems in category learning, and examining their effect on the correlation between working memory span - a measure of the control of executive attentionand overall learning accuracy. These two indicators were the use of more than one type of representation and taskswitch cost. Subjects who used more than one category bound type, and subjects who had greater task-switch cost, showed a greater correlation between working memory span and learning accuracy; moreover, the two indicator variables were correlated. Subjects who were identified as using more than one category bound type showed greater task-switch costs than those who used just one.

These findings are consistent with the relationships illustrated in Figure 1. That figure explains these relationships as being driven by the use of stimulus-dependent representation. As was described previously, when people partition a category learning task into subtasks, they need to learn to identify which subtask goes with which stimuli. Each subtask depends on a different category representation.

The present results can be accounted for at a conceptual level by theories of category learning, such as ATRIUM, that incorporate stimulus-dependent representation. No

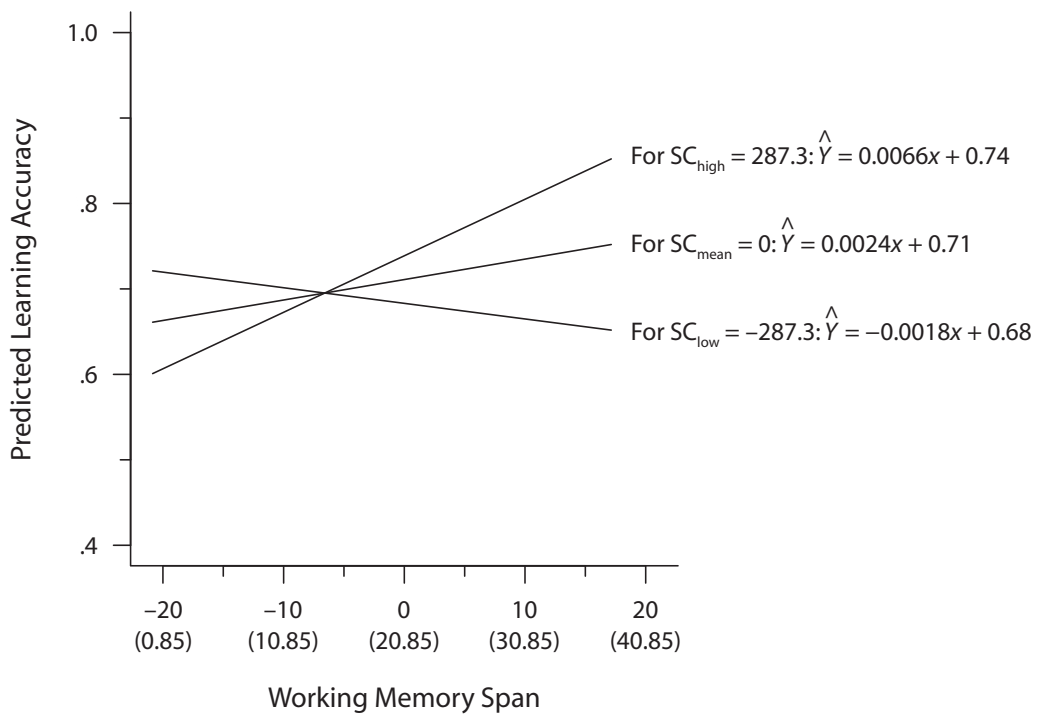

Figure 5. Regression of learning accuracy $(Y)$ on working memory span $(x)$ at three levels of switch cost. Simple regression equations are for centered data. Working memory spans shown in parentheses indicate uncentered values. 
current model, however, can provide a full computational account of the data. Although ATRIUM holds that categorization can be construed as involving task switching, it does not currently predict RTs, nor does it have any mechanism that has been identified as representing executive attention. To account for RTs would require the inclusion of principles in the model to govern its dynamics. Three additional principles would likely be sufficient to allow ATRIUM to account for task-switching costs manifested in subjects' RTs. The first of these principles is that activation builds up over time; the second is that it takes time for activations to decay back to a resting state in the absence of input; and the third is that activation for one potential response must exceed that of the other responses by some amount for the response to be generated. (See Usher \& McClelland, 2001, for an examination of these kinds of principles in an RT model.) Given these principles, the model would generate responses more quickly on the second of two consecutive trials that use the same representation. This is because, on the second trial, residual activation from the first in the gating mechanism would allow the selected representation to influence the output more quickly, and this would lead to faster responses. Likewise, on a trial that requires a switch from one representation to another, it would take time for the gating mechanism to allow activation from the other representation to begin to influence the output, and this would delay the model's response. Under this framework, the gating mechanism can be thought of as retrieving task goals based on the current input, as well as reprogramming the attentional system to execute the goals.

The most likely locus for an account of executive attention in ATRIUM would also be in the gating mechanism. The gating mechanism learns to select the best representation for each stimulus over time via a connectionist network. The activations of the output nodes in this network are currently mapped to the probability of selecting representation $r$ using the function

$$
p_{r}=\frac{\exp \left(\phi_{g} g_{r}+\beta_{r}\right)}{\sum_{R} \exp \left(\phi_{g} g_{R}+\beta_{R}\right)},
$$

where $\mathrm{g}_{r}$ is the activation of the gate node for representation $r, \beta_{r}$ is bias to use representation $r$, and $\phi_{g}>0$ is the gain of the gating mechanism, which determines how extreme the probabilities tend to be. If gating mechanism gain, $\phi_{g}$, has a low value, the probabilities hover near (1/number of representations), meaning that no single representation is likely to be favored on any trial. As the gain increases, the probabilities become more extreme, with one probability being pushed toward 1, and the other probabilities being pushed toward 0 . Furthermore, when the gain is low, the model's responses are not systematically driven by any single representation. When the gain is high, a single representation will tend to dominate on each trial. When the model is using multiple representations, the gain will tend to have a greater impact on predicted accuracy, because the model needs to shift sharply from representation to representation, depending on the current stimulus to make correct responses. When the model is using a single representation, the gating mechanism can be pushed to use a single representation by the bias parameter for that representation, $\beta_{r}$. Although the gain will still influence the model's accuracy, it becomes much less important. In a dynamical version of ATRIUM that incorporates the principles outlined by Usher and McClelland (2001), the strength of lateral inhibition should have an effect similar to that of the gain.

Although all the results of this study can be seen as consistent with theories of category learning that posit stimulus-dependent representation, they are inconsistent with most other models of category learning - both single- and multiple-system theories. Single-system theories of category learning, such as the generalized context model (GCM; Nosofsky, 1986), ALCOVE (Kruschke, 1992), SUSTAIN (Love, Medin, \& Gureckis, 2004), and the exemplar-based random-walk model (EBRW; Nosofsky \& Palmeri, 1997), cannot learn to partition a task into subtasks, because they are limited to a single categorylearning approach and a single representation. Some who support these theories have argued that, in fact, people do not partition category-learning tasks among multiple representations, but that extensions to single-system theories are sufficient to account for people's patterns of classification (Nosofsky \& Johansen, 2000; Rodrigues \& Murre, 2007). Even if these theories can account for classification data, they contain no principles that would allow them to predict differential task-switching costs, the relationship between executive attention and learning performance among the subset of subjects who appeared most likely to use multiple representations, or the relationship between task-switching costs and working memory. According to these single-system theories, both category substructures in the present study should be represented in the same way and with the same attentional allocation. Thus, they most likely predict that a switch from one category substructure to another should take the same amount of time for all subjects; and similarly, that executive attention should play the same role for all subjects.

Multiple-system theories of category learning are better suited to account for the present results - with one important exception. Whereas theories of category learning that allow for task partitioning can learn to use multiple representations within a single learning task, other multiplesystem theories posit that category learning consists of learning which system is best suited to learn the task as a whole. For example, COVIS (Ashby et al., 1998) is designed so that two category learning systems compete to learn the task at hand. For a given stimulus, the system that can make the less equivocal decision wins the competition; over time, COVIS learns to favor the system that has won the most overall. Similarly, Anderson and Betz (2001) used the ACT-R framework to create a hybrid model of category learning that uses the overall success rates for each component to select whether its rule- or similarity-based mechanism is responsible for generating each response. Even though these theories have multiple representations available, the representation they use on each trial is based on their overall history of success and, by the end of training, is independent of the current input. 
Thus, like single-system theories of category learning, they would need to account for the present results using a single system for the stimuli from both substructures and would encounter the same explanatory problems. Nevertheless, these theories could possibly be extended in a straightforward way to incorporate stimulus-dependent representation. This would allow them to account directly for the data from the present study.

One limitation of the methodology used in the present study was that the classification of subjects as using one or two types of category bounds did not seem to correspond perfectly with the use of stimulus-dependent representation. The classification of subjects as utilizing one or two representations was based on the architecture of ATRIUM. As ATRIUM has been described by Erickson and Kruschke (e.g., Erickson, 1999; Erickson \& Kruschke, 2002), it is composed of a single similarity-based module and one rule-based module per input dimension. Yang and Lewandowsky (2004), however, have successfully used a version of ATRIUM with two aligned rule modules to account for category learning results from a category structure composed of two substructures with parallel category boundaries. ${ }^{4}$ This allowed the model to account for the results of studies in which subjects showed evidence of partitioning in a category learning task that could be decomposed into two subtasks with aligned category boundaries. The finding in the present study that some subjects who used a single bound type showed RT costs when switching from one substructure to another provides support for Yang and Lewandowsky's (2004) modification of ATRIUM's original structure. This suggests that the assay used to distinguish between subjects who used one and two representations might have had too strict a criterion for inclusion in the two-representation group. An empirical remedy for this problem is suggested in the work of Yang and Lewandowsky $(2003,2004)$. They identified subjects as having partitioned their task into subtasks through an analysis of transfer trials in which the stimuli provided potentially contradictory information. For these stimuli, the color cue indicated one substructure, whereas the other stimulus attributes (e.g., rectangle height and segment position) indicated the other substructure. Subjects who gave responses that indicated greater sensitivity to the color cue were classified as having partitioned the task. Although this kind of analysis still provides only an indirect measure of subjects' underlying strategy, it could afford a more accurate division between the groups of subjects. Nevertheless, even though the standard for inclusion in the two-representation group that was used in the present study may have been too conservative, this limitation does not provide an alternate explanation for the results. Instead, it suggests that the true number-ofrepresentations $\times$ substructure-switch interaction shown in Figure 4 may be even larger than what was measured.

This article advances two new sources of evidence for multiple-system theories of category learning. First, when subjects successfully partition a category learning task into subtasks, their RTs reflect costs of switching from one subtask to another. Second, executive attention appears to play a more important role in learning for these same sub- jects than for other subjects who tended not to partition the learning task. Both of these sources of evidence appear to be problematic for theories of category learning that lack stimulus-dependent representation. This includes singlesystem theories of category learning, as well as multiplesystem theories that seek to identify the single system best suited for an overall category-learning task. The data are, however, consistent with category learning frameworks, such as ATRIUM, in which people are presumed to be able to learn to use multiple representations within a single category-learning task.

\section{AUTHOR NOTE}

Preparation of this article was supported by grants provided by the Academic Senate of the University of California, Riverside. I thank Robert Rosenthal and Daniel J. Ozer for their help developing the analyses in this article. I thank Jesse Breneman for his contributions on preliminary studies. Correspondence concerning this article should be addressed to M. A. Erickson, Department of Psychology, University of California, Riverside, CA 92521 (e-mail: erickson@ucr.edu).

\section{REFERENCES}

AKaIKe, H. (1974). A new look at the statistical model identification. IEEE Transactions in Automatic Control, 19, 716-723.

Anderson, J. R., \& Betz, J. (2001). A hybrid model of categorization. Psychonomic Bulletin \& Review, 8, 629-647.

Ashby, F. G., Alfonso-Reese, L. A., Turken, A. U., \& Waldron, E. M. (1998). A neuropsychological theory of multiple systems in category learning. Psychological Review, 105, 442-481.

Ashby, F. G., Ell, S. W., \& Waldron, E. M. (2003). Procedural learning in perceptual categorization. Memory \& Cognition, 31, 1114-1125.

AshBy, F. G., \& GoTT, R. E. (1988). Decision rules in the perception and categorization of multidimensional stimuli. Journal of Experimental Psychology: Learning, Memory, \& Cognition, 14, 33-53.

Ashby, F. G., \& Townsend, J. T. (1986). Varieties of perceptual independence. Psychological Review, 93, 154-179.

Cohen, J., Cohen, P., West, S. G., \& Aiken, L. S. (2003). Applied multiple regression/correlation analysis for the behavioral sciences (3rd ed.). Mahwah, NJ: Erlbaum.

Conway, A. R. A., Cowan, N., \& Bunting, M. F. (2001). The cocktail party phenomenon revisited: The importance of working memory capacity. Psychonomic Bulletin \& Review, 8, 331-335.

Conway, A. R. A., Cowan, N., Bunting, M. F., Therriault, D. J., \& MinkofF, S. R. B. (2002). A latent variable analysis of working memory capacity, short-term memory capacity, processing speed, and general fluid intelligence. Intelligence, 30, 163-183.

Daneman, M., \& CARPEnTER, P. A. (1980). Individual differences in working memory and reading. Journal of Verbal Learning \& Verbal Behavior, 19, 450-466.

Daneman, M., \& Carpenter, P. A. (1983). Individual differences in integrating information between and within sentences. Journal of Experimental Psychology: Learning, Memory, \& Cognition, 9, 561-583.

DANEMAN, M., \& GREen, I. (1986). Individual differences in comprehending and producing words in context. Journal of Memory \& Language, 25, 1-18.

Denton, S. E., Kruschke, J. K., \& Erickson, M. A. (in press). Rulebased extrapolation: A continuing challenge for exemplar models. Psychonomic Bulletin \& Review.

ENGLE, R. W. (2002). Working memory capacity as executive attention. Current Directions in Psychological Science, 11, 19-23.

ENGLE, R. W. (2005). Automated symmetry span (E-Prime) [Computer software]. Retrieved from psychology.gatech.edu/renglelab/tasks.htm.

Engle, R. W., \& Kane, M. J. (2004). Executive attention, working memory capacity, and a two-factor theory of cognitive control. In B. H. Ross (Ed.), The psychology of learning and motivation: Advances in research and theory (Vol. 44, pp. 145-199). San Diego: Academic Press.

Engle, R. W., Tuholski, S. W., Laughlin, J. E., \& Conway, A. R. A. 
(1999). Working memory, short-term memory, and general fluid intelligence: A latent-variable approach. Journal of Experimental Psychology: General, 128, 309-331.

ErICKSON, M. A. (1999). Rules and exemplars in category learning (Doctoral dissertation, Indiana University, Bloomington). Dissertation Abstracts International, 60, 2377B.

Erickson, M. A., \& KruschKe, J. K. (1998). Rules and exemplars in category learning. Journal of Experimental Psychology: General, 127, 107-140.

Erickson, M. A., \& KruschKe, J. K. (2002). Rule-based extrapolation in perceptual categorization. Psychonomic Bulletin \& Review, 9, $160-168$.

Green, D. M., \& Swets, J. A. (1966). Signal detection theory and psychophysics. Oxford: Wiley.

Kalish, M. L., Lewandowsky, S., \& Kruschke, J. K. (2004). Population of linear experts: Knowledge partitioning and function learning. Psychological Review, 111, 1072-1099.

Kane, M. J., Bleckley, M. K., Conway, A. R. A., \& Engle, R. W. (2001). A controlled-attention view of working-memory capacity. Journal of Experimental Psychology: General, 130, 169-183.

KANE, M. J., \& ENGLE, R. W. (2002). The role of prefrontal cortex in working memory capacity, executive attention, and general fluid intelligence: An individual-differences perspective. Psychonomic Bulletin \& Review, 9, 637-671

KANE, M. J., \& ENGLE, R. W. (2003). Working-memory capacity and the control of attention: The contributions of goal neglect, response competition, and task set to Stroop interference. Journal of Experimental Psychology: General, 132, 47-70.

Kane, M. J., Hambrick, D. Z., Tuholski, S. W., Wilhelm, O., Payne, T. W., \& ENGLE, R. W. (2004). The generality of working memory capacity: A latent-variable approach to verbal and visuospatial memory span and reasoning. Journal of Experimental Psychology: General, 133, 189-217.

KruschKe, J. K. (1992). ALCOVE: An exemplar-based connectionist model of category learning. Psychological Review, 99, 22-44.

KruschKe, J. K., \& ERICKSON, M. A. (1994). Learning of rules that have high-frequency exceptions: New empirical data and a hybrid connectionist model. In Proceedings of the Sixteenth Annual Conference of the Cognitive Science Society (pp. 514-519). Hillsdale, NJ: Erlbaum.

Lewandowsky, S., Kalish, M. L., \& NGang, S. K. (2002). Simplified learning in complex situations: Knowledge partitioning in function learning. Journal of Experimental Psychology: General, 131, 163-193.

LEWANDOWSKy, S., \& KIRSNER, K. (2000). Knowledge partitioning: Context-dependent use of expertise. Memory \& Cognition, 28, 295-305.

Lewandowsky, S., Roberts, L., \& YAnG, L.-X. (2006). Knowledge partitioning in categorization: Boundary conditions. Memory \& Cognition, 34, 1676-1688.

LoGAN, G. D. (2004). Working memory, task switching, and executive control in the task span procedure. Journal of Experimental Psychology: General, 133, 218-236.

Logan, G. D., \& Gordon, R. D. (2001). Executive control of visual attention in dual-task situations. Psychological Review, 108, 393-434.

Long, D. L., \& PRAT, C. S. (2002). Working memory and Stroop interference: An individual differences investigation. Memory \& Cognition, 30, 294-301

Love, B. C., Medin, D. L., \& Gureckis, T. M. (2004). SUSTAIN: A network model of category learning. Psychological Review, 111, 309-332.

Meng, X., Rosenthal, R., \& Rubin, D. B. (1992). Comparing correlated correlation coefficients. Psychological Bulletin, 111, 172-175.

Monsell, S. (2003). Task switching. Trends in Cognitive Sciences, 7, 134-140.
Norman, A. D., \& Shallice, T. (1986). Attention to action: Willed and automatic control of behavior. In R. J. Davidson, G. E. Schwartz, \& D. E. Shapiro (Eds.), Consciousness and self-regulation: Advances in research (Vol. 4, pp. 1-18). New York: Plenum.

Nosofsky, R. M. (1986). Attention, similarity, and the identificationcategorization relationship. Journal of Experimental Psychology: General, 115, 39-57.

Nosofsky, R. M., \& Johansen, M. K. (2000). Exemplar-based accounts of "multiple-system" phenomena in perceptual categorization. Psychonomic Bulletin \& Review, 7, 375-402.

Nosofsky, R. M., \& PALMERI, T. J. (1997). An exemplar-based random walk model of speeded classification. Psychological Review, 104, 266-300.

Rodrigues, P. M., \& Murre, J. M. J. (2007). Rules-plus-exception tasks: A problem for exemplar models? Psychonomic Bulletin \& Review, 14, 640-646.

Rosenthal, R. (1991). Meta-analytic procedures for social research (Rev. ed.). Thousand Oaks, CA: Sage.

Rosenthal, R., \& Rosnow, R. L. (1991). Essentials of behavioral research: Methods and data analysis (2nd ed.). New York: McGraw-Hill.

Schwarz, G. (1978). Estimating the dimension of a model. Annals of Statistics, 6, 461-464.

SHute, V. J. (1991). Who is likely to acquire programming skills? Journal of Educational Computing Research, 7, 1-24.

Smith, E. E., Patalano, A. L., \& Jonides, J. (1998). Alternative strategies of categorization. Cognition, 65, 167-196.

Stroop, J. R. (1935). Studies of interference in serial verbal reactions. Journal of Experimental Psychology, 18, 643-662.

Turner, M. L., \& ENGLe, R. W. (1989). Is working memory capacity task dependent? Journal of Memory \& Language, 28, 127-154.

UNDERWOOD, B. J. (1975). Individual differences as a crucible in theory construction. American Psychologist, 30, 128-134.

Usher, M., \& MCClelland, J. L. (2001). The time course of perceptual choice: The leaky, competing accumulator model. Psychological Review, 108, 550-592.

YANG, L.-X., \& LewANDowsKy, S. (2003). Context-gated knowledge partitioning in categorization. Journal of Experimental Psychology: Learning, Memory, \& Cognition, 29, 663-679.

YANG, L.-X., \& LEWANDOWSKY, S. (2004). Knowledge partitioning in categorization: Constraints on exemplar models. Journal of Experimental Psychology: Learning, Memory, \& Cognition, 30, 1045-1064.

\section{NOTES}

1. Atrium, attention to rules and instances in a unified model. 2. Fits were also compared using the BIC (Schwarz, 1978). Under the BIC, $42 / 170=25 \%$ of the subjects changed fit categories relative to the AIC. Nevertheless, the subsequent qualitative findings remained the same. Therefore, only the results based on the AIC classifications are presented.

3. Mean correlations were computed after applying Fisher's $z$ transformation $z_{r}=1 / 2 \log [(1+r) /(1-r)]$ to each correlation. The reported values were then computed by applying the inverse transformation to the mean $z_{r} \mathrm{~s}$ (Rosenthal \& Rosnow, 1991).

4. The rule modules used by Yang and Lewandowsky (2004) also had boundaries that were not perpendicular to the hypothesized stimulus dimensions. This is an additional variation from the original formulation of ATRIUM that is currently under investigation.

(Manuscript received May 2, 2007; revision accepted for publication November 9, 2007.) 